\title{
ON A GEOMETRIC PROPERTY OF THE SET OF INVARIANT MEANS ON A GROUP
}

\author{
CHING CHOU
}

\begin{abstract}
Abstracr. If $G$ is a discrete group and $x \in G$ then $x \sim$ denotes the homeomorphism of $\beta G$ onto $\beta G$ induced by left multiplication by $x$. A subset $K$ of $\beta G$ is said to be invariant if it is closed, nonempty and $x \sim K \subset K$ for each $x \in G$. Let $M L(G)$ denote the set of left invariant means on $G$. (They can be considered as measures on $\beta G$.)

THEOREM. Let $G$ be a countably infinite amenable group and let $K$ be an invariant subset of $\beta G$. Then the nonempty $w^{*}$-compact convex set $M(G, K)=\{\phi \in M L(G)$ : suppt $\phi \subset K\}$ has no exposed points (with respect to $w^{*}$-topology). Therefore, it is infinite dimensional.
\end{abstract}

1. Let $S$ be a semigroup, $m(S)$ the Banach space of bounded real functions on $S$ with the sup norm $\phi \in m(S)^{*}$ is called a mean if $\|\phi\|=1$ and $\phi(f) \geqq 0$ for $f \geqq 0$. Let $\beta S$ denote the Stone-Čech compactification of the discrete set $S$. Each $f \in m(S)$ can be extended to a continuous function on $\beta S$. The extended function will again be denoted by $f$. If $\phi$ is a mean on $m(S)$ then $\mu_{\phi}$ will denote the probability measure on $\beta S$ defined by $\int_{\beta S} f d \mu_{\phi}=\phi(f), f \in m(S)$.

A mean $\phi \in m(S) *$ is said to be left invariant if $\phi(f)=\phi\left(l_{s} f\right)$ for $f \in m(S)$ and $s \in S$ where $l_{s} f \in m(S)$ is defined by $\left(l_{s} f\right)\left(s_{1}\right)=f\left(s s_{1}\right)$. Denote the set of all left invariant means on $S$ by $M L(S)$. If $M L(S)$ is nonempty then we say $S$ is left amenable. In this case, $M L(S)$ is $w^{*}$-compact convex (cf. [5]).

For $s \in S, s^{\sim}$ denotes the continuous mapping of $\beta S$ into itself defined by $s^{\sim} s_{1}=s s_{1}, s_{1} \in S$. A subset $K$ of $\beta S$ is said to be invariant if it is nonempty, closed and $s^{\sim} K \subset K$ for each $s \in S$. If $K$ is an invariant subset of $\beta S$, set $M(S, K)=\left\{\phi \in M L(S)\right.$ : suppt $\left.\mu_{\phi} \subset K\right\}$.

If $S$ is left amenable and $K$ is an invariant subset of $\beta S$ then, by Day's fixed point theorem [6], $M(S, K)$ is nonempty. It is also easy to check that $M(S, K)$ is $w^{*}$-compact convex and each extreme point of $M(S, K)$ is also an extreme point of $M L(S)$. Thus, by the Kreĭn-Milman theorem, $M(S, K)$ contains at least one extreme point of $M L(S)$. In general $\beta S$ contains many mutually disjoint invariant sets. For example, when $S$ is an infinite amenable group then $\beta S$

Received by the editors December 21, 1970.

AMS 1970 subject classifications. Primary 43A07, 46N05, 22D40.

Key words and phrases. Invariant means, amenable groups, mean ergodic theorem, exposed points, Stone-Čech compactification.

Copyright @ 1971, American Mathematical Society 
has at least $2^{c}$ such sets (cf. [2]). Here $c$ denotes the cardinality of the continuum. Therefore, we concluded in [2] that for each infinite amenable group $S$ the set $M L(S)$ has at least $2^{c}$ extreme points. It is then natural to ask whether $M L(S)$ has any exposed points (with respect to the $w^{*}$-topology). Cf. [10] for the definition of exposed points.

Indeed, if $S$ is a left amenable semigroup and $K$ an invariant subset of $\beta S$ one may ask the following more general questions: How big is the set $M(S, K)$ ? Does it have any exposed points? Raimi [12] proved that for each invariant subset $K$ of $\beta N, N$ the additive semigroup of positive integers, $M(N, K)$ has at least two extreme points. Recently, Fairchild [7], adapting the technique in Granirer [8], proved that if $S$ is a left amenable, countably infinite cancellation semigroup and $K$ is an invariant subset of $\beta S$ then $M(S, K)$ has infinitely many extreme points. The main result of this paper is the following.

Theorem. Let $G$ be a countably infinite amenable group and $K$ be an invariant subset of $\beta G$. Then $M(G, K)$ has no exposed points.

Since every compact convex subset of a finite-dimensional topological vector space has exposed points, the above seemingly negative result implies, among other things, Fairchild's result which we mentioned above.

If $X$ is a discrete set and $\omega \in \beta X$ then $\omega^{\prime}$ will denote the element in $m(X)^{*}$ defined by $\omega^{\prime}(f)=f(\omega), f \in m(X)$. If $A$ and $B$ are sets, $A \triangle B$ $=(A \backslash B) \cup(B \backslash A)$ and $|A|=$ number of elements in $A$.

2. Throughout this section $G$ denotes a countably infinite amenable group with a fixed sequence of finite subsets, $F_{n}$, such that

(F1) $F_{n} \subset F_{n+1}, n=1,2, \cdots ; \cup F_{n}=G$,

(F2) $F_{n}=F_{n}^{-1}, n=1,2, \cdots$,

(F3) $\lim _{n}\left|x F_{n} \triangle F_{n}\right| /\left|F_{n}\right|=0, x \in X$.

The existence of such a sequence $F_{n}$ for every countable amenable group is proved by Namioka [11].

For a positive integer $n, T_{n}: m(G) \rightarrow m(G)$ is defined by

$$
\left(T_{n} f\right)(\omega)=\left(1 /\left|F_{n}\right|\right) \sum_{x \in F_{n}} f\left(x^{\sim} w\right), \quad f \in m(G), w \in \beta G .
$$

Note that $\left(T_{n} f\right)(\omega)=\left(T_{n}^{*} \omega^{\prime}\right) f$. For $w \in \beta G$, set $Q_{\omega}=$ the set of $w^{*}$. cluster points of the sequence $T_{n}^{*} \omega^{\prime}$.

Lemma 1. Let $K$ be an invariant subset of $\beta G$ and $\omega \in K$. Then $Q_{\omega} \subset M(G, K)$. 
Proof. Let $\phi=\lim _{\alpha} T_{n_{\alpha}}^{*} \omega^{\prime} \in Q_{\omega}$. Then, for $f \in m(G)$ and $x \in G$,

$$
\begin{aligned}
\mid\left(T_{n_{\alpha}}^{*} \omega^{\prime}\right) f & -\left(T_{n_{\alpha}}^{*} \omega^{\prime}\right) l_{x} f \mid \\
& =\left(1 /\left|F_{n_{\alpha}}\right|\right)\left|\sum_{y \in F_{n_{\alpha}}} f\left(y^{\sim} \omega\right)-\sum_{y \in F_{n_{\alpha}}} f\left(x^{\sim} y^{\sim} \omega\right)\right| \\
& \leqq\left(\left|F_{n_{\alpha}} \triangle x F_{n_{\alpha}}\right| /\left|F_{n_{\alpha}}\right|\right) \cdot\|f\| \rightarrow 0 \quad \text { as } n_{\alpha} \rightarrow \infty, \quad \text { by (F3). }
\end{aligned}
$$

Thus $\phi\left(l_{x} f\right)=\phi(f)$, i.e., $\phi \in M L(G)$. The fact that suppt $M_{\phi} \subset K$ is obviously true.

Lemma 2. Let $\omega_{n}$ be a sequence of distinct elements in $\beta X$ where $X$ is an infinite discrete set. Let $n_{1}<n_{2}<\cdots$ be an increasing sequence of positive integers. Then the sequence

$$
\left(1 / n_{j}\right)\left(\omega_{1}^{\prime}+\omega_{2}^{\prime}+\cdots+\omega_{n_{j}}^{\prime}\right), \quad j=1,2, \cdots,
$$

is not convergent in the $w^{*}$-topology and hence has at least two w*-cluster points.

Proof. When $n_{j}=j$ this lemma is the main theorem of Rudin [13]. His proof also works for this slightly generalized proposition.

The following mean ergodic theorem is similar to that of Calderón [1] and Tempel'man [14]. If $B$ is a Banach space, $\mathcal{L}(B)$ will denote the algebra of bounded linear operators from $B$ in to itself.

LEMMA 3. Let $B$ be a Banach space and $u$ be a mapping of $G$ into $\mathfrak{L}(B)$ such that (1) $\mathfrak{u}^{x y}=\mathfrak{u}^{y} \mathfrak{u}^{x}, x, y \in G,(2)\left\|\mathfrak{u}^{x}\right\| \leqq C, a$ constant, for all $x \in G$. Assume $A \subset B$ is weakly compact and $\mathcal{u}^{x} A \subset A$ for $x \in G$. Then for each $f \in A$ the sequence $P_{n}(f)=\left(1 /\left|F_{n}\right|\right) \sum_{x \in F_{n}} u^{x} f$ converges in norm.

Proof. The proof is similar to that of [1]. We only give a sketch here. Choose a sequence $n_{j}$ such that $P_{n_{j}}(f)$ converges to an element $f_{0} \in A$ is weak topology. Note that we have the following two inequalities: For $x \in G$,

$$
\begin{aligned}
\left\|\mathcal{u}^{x}\left(P_{n} f\right)-P_{n} f\right\| & =\left(1 /\left|F_{n}\right|\right) \| \sum_{y \in F_{n}} \mathcal{u}^{y x} f-\sum_{y \in F_{n}} \text { uvf }^{y} \| \\
& \leqq\left(C\left|F_{n} x \Delta F_{n}\right| /\left|F_{n}\right|\right) \cdot\|f\| \\
& =\left(C\left|x^{-1} F_{n} \triangle F_{n}\right| /\left|F_{n}\right|\right) \cdot\|f\| \\
\left\|P_{n}\left(u^{*} f\right)-P_{n} f\right\| & \leqq\left(C\left|x F_{n} \triangle F_{n}\right| /\left|F_{n}\right|\right) \cdot\|f\| .
\end{aligned}
$$

By (F3) and the above two inequalities, $\lim _{n}\left\|\mathcal{u}^{x}\left(P_{n} f\right)-P_{n} f\right\|=0$ and 
$\lim _{n}\left\|P_{n}\left(\mathcal{u}^{x} f\right)-P_{n} f\right\|=0(x \in G)$. Using these two equalities, it is not difficult to conclude that $\lim P_{n} f=f_{0}$ in norm.

Clearly the above lemma also holds if we change (1) into (1)': $\mathcal{u}^{x y}=\mathfrak{u}^{x} u^{y}, x, y \in G$.

The following lemma is a refinement of [9, Theorem 4].

Lemma 4. Let $K$ be an invariant subset of $\beta G$. Then for each $f \in m(G)$ there exist $\omega \in K$ and $\phi_{i} \in Q_{\omega}, i=1,2, \phi_{1} \neq \phi_{2}$, such that

$$
\sup \{\phi(f): \phi \in M(G, K)\}=\phi_{1}(f)=\phi_{2}(f) .
$$

Proof. Denote $\sup \{\phi(f): \phi \in M(G, K)\}$ by $\alpha(f)$. Since $M(G, K)$ is nonempty and $w^{*}$-compact there exists $\phi \in M(G, K)$ such that $\phi(f)=\alpha(f)$. Let $B=L^{2}\left(\mu_{\phi}\right)$ and $\mathcal{U}^{x}$ be defined by $\left(\mathcal{u}^{x} h\right)(\omega)=h\left(x^{\sim} \omega\right)$, $h \in L^{2}\left(\mu_{\phi}\right), \omega \in K$. Since $\phi$ is left invariant, $\left\|u^{x}\right\|=1, x \in G$, and clearly $\mathfrak{U}^{x y}=\mathfrak{u}^{y u x}$. Also note that, if $f \in m(G)$,

$$
\left(P_{n} f\right)(\omega)=\left(1 /\left|F_{n}\right|\right) \sum_{y \in F_{n}} f(y \sim \omega)=\left(T_{n} f\right)(\omega), \quad \omega \in K .
$$

Therefore, by Lemma $3, \lim _{n} T_{n} f$ exists in $L^{2}\left(\mu_{\phi}\right)$ norm. Denote the limit by $f_{0}$. Choose a subsequence $n_{j}$ such that $\lim _{j}\left(T_{n_{j}} f\right)(\omega)=f_{0}(\omega)$ exists for $\omega \in D \subset K$ where $D$ is a Borel subset of $K$ and $\mu_{\phi}(D)=1$. Thus

$$
\int f_{0} d \mu_{\phi}=\lim _{j} \int\left(T_{n_{j}} f\right) d \mu_{\phi}=\phi\left(T_{n_{j}} f\right)=\phi(f) .
$$

Also note that if $\omega \in D$ then there exists $\psi \in Q_{\omega}$ such that $\psi(f)=f_{0}(\omega)$. Hence by Lemma 1

$$
f_{0}(\omega) \leqq \alpha(f)=\phi(f)
$$

Compare (i) and (ii) we see that $f_{0}(\omega)=\phi(f)$ for almost all $\omega \in D$. In particular, there exists $\omega_{0} \in K$ such that

$$
\phi(f)=\lim _{j}\left(T_{n_{j}} f\right)\left(\omega_{0}\right) .
$$

Finally, note that if $x, y \in G, x \neq y$, then $x \sim \omega_{0} \neq y \sim \omega_{0}[3$, Lemma 1]. Thus we may apply Lemma 2 to the set $\left\{x \sim \omega_{0}: x \in G\right\}$ and conclude that the sequence

$$
\left(1 /\left|F_{n_{j}}\right|\right) \sum_{x \in F_{n}}\left(x^{\sim} \omega_{0}\right)^{\prime}=T_{n_{j} \omega^{\prime}}^{*}
$$

has at least two $\omega^{*}$-cluster points $\phi_{1}$ and $\phi_{2}$. By the definition, $\phi_{i}$ $\in Q_{\omega_{0}}, i=1,2$. That $\phi_{1}(f)=\phi_{2}(f)=\alpha(f)$ follows directly from (iii). 
THEOREM 1. Let $G$ be a countably infinite amenable group and let $K$ be an invariant subset of $\beta G$. Then the w*-compact convex set $M(G, K)$ has no exposed points.

Proof. $\phi \in M(G, K)$ is an exposed point if and only if there exists $f \in M(G)$ such that $\phi(f)>\psi(f)$ for $\psi \in M(G, K), \psi \neq \phi$. By Lemma 4, there is no $\phi \in M(G, K)$ which has this property.

When $K=\beta G, M(G, K)=M L(G)$. We want to state this special case separately.

Corollary 1. Let $G$ be a countably infinite amenable group. Then $M L(G)$ has exactly $2^{c}$ extreme points but has no exposed points.

That $M L(G)$ has $2^{c}$ extreme points is contained in [2].

COROLlaRy 2. Let $S$ be a left amenable countably infinite cancellation semigroup.

(1) If $K$ is a minimal invariant subset of $\beta S$ then $M(S, K)$ has no exposed points.

(2) If $K$ is an invariant subset of $\beta S$ then $M(S, K)$ cannot be embedded into a Banach space affinely and topologically. In particular, $M(S, K)$ has to be infinite dimensional.

Proof. (1) Since $S$ is cancellative it can be considered as a subsemigroup of an amenable group $G$ (cf. [15]). We may assume that $G$ is generated by $S$. In particular, $G$ is also countably infinite. Let $K$ be a minimal invariant subset of $\beta S$. Fix any $\phi \in M(S, K)$. For $s \in S$, using the fact that $s^{\sim}$ is one-one [2, Lemma 2.1], one gets that $\mu_{\phi}(s \sim K)=\mu_{\phi}(K)=1$. Therefore suppt $\mu_{\phi} \subset s \sim K \subset K$. On the other hand, since suppt $\mu_{\phi}$ is invariant [15, Theorem 4.3] and $K$ is minimal invariant we conclude that $K=s^{\sim} K=\operatorname{suppt} \mu_{\phi}$. Since $G$ is generated by $S$ we see that $x^{\sim} K=K$ for each $x \in G$, i.e., $K$ is an invariant subset of $\beta G$. Therefore, by Theorem $1, M(G, K)$ has no exposed points. It is easily checked that $M(G, K)=M(S, K)$. Thus $M(S, K)$ has no exposed points.

(2) Let $K$ be an invariant subset of $\beta S$. By Zorn's Lemma, $K$ contains a minimal invariant subset $K_{1}$. Hence, by (1), $M\left(S, K_{1}\right)$ has no exposed points. Note that $M\left(S, K_{1}\right) \subset M(S, K)$. Thus the result follows from the well-known fact that every compact convex subset of a Banach space has exposed points (cf. Klee [10]).

Let $G$ be an amenable group and $H$ a homomorphic image of $G$. Then it is known that if, for each invariant subset $K_{1}$ of $\beta H, M\left(H, K_{1}\right)$ is infinite dimensional then $M(G, K)$ is also infinite dimensional for 
each invariant subset $K$ of $\beta G$ [7, Proposition 5.7]. In particular, if $G$ is an infinite abelian group then $G$ has a countably infinite homomorphic image $H$ [13]. Thus by Corollary 2 we have the following.

Corollary 3. Let $S$ be an infinite abelian cancellation semigroup and $K$ an invariant subset of $\beta S$. Then $M(S, K)$ is infinite dimensional.

Another consequence of Lemma 4 is the following generalization of Theorem 4 in [9].

THEOREM 2. Let $G$ be a countable amenable group with a sequence of finite sets $F_{n}$ which satisfies (F1), (F2) and (F3). Then $M L(G)$ equals the $\omega^{*}$-closed convex hull of $\cup\left\{Q_{\omega}: \omega \in \beta G\right\}$.

3. Remarks. (1) We believe that Theorem 1 holds for every infinite amenable group. But we do not know how to prove it.

When $G$ is a countably infinite amenable group and $K$ an invariant subset of $\beta G$ then Theorem 1 tells us that $M(G, K)$ has infinitely many extreme points. It is interesting to know exactly how many extreme points are in $M(G, K)$. Are there $2^{c}$ of them?

(2) Let $G$ be a unimodular $\sigma$-compact locally compact amenable group. Then, same as the discrete case, there exists a sequence $F_{n}$ of compact neighborhoods of the identity such that (F1), (F2) and (F3) hold [4, Theorem 4]. Of course, here in (F3), $|A|$ denotes the Haar measure of a set $A$, instead of the number of elements in $A$. The mean ergodic theorem (Lemma 3 ) also holds for the above $G$ and $F_{n}$ : Let $x \rightarrow \mathcal{U}^{x}$ be a weakly continuous homomorphism of $G$ into $\mathscr{L}(B), B$ a Banach space, such that $\left\|u^{x}\right\| \leqq C$ for each $x \in G$ where $C$ is a fixed constant. Suppose there is a weakly compact convex set $A \subset B$ such that $u^{x} A \subset A, x \in G$. Then for each $u \in A,\left(1 /\left|F_{n}\right|\right) \int_{F_{n}} \mathcal{u}^{x}(u) d x$ converges in norm to an element in $A$ (cf. Calderón [1] and Tempel'man [14]).

[1] and [14] also contain an individual ergodic theorem with respect to a sequence similar to $F_{n}$ above with an additional condition: there exists $k>0$ such that

$$
\left|F_{n}^{2}\right| \leqq k\left|F_{n}\right|, \quad n=1,2, \cdots
$$

We do not know whether the individual ergodic theorem holds without (E). Even for a countable amenable discrete group it is unlikely in general that a sequence $F_{n}$ can be found to satisfy (F1), (F2), (F3) and $(\mathrm{E})$ simultaneously. 


\section{REFERENCES}

1. A. P. Calderón, A general ergodic theorem, Ann. of Math. (2) 58 (1953), 182191. MR 14, 1071.

2. C. Chou, On the size of the set of left invariant means on a semigroup, Proc. Amer. Math. Soc. 23 (1969), 199-205. MR $40 \# 710$.

3. - On a conjecture of $E$. Granirer concerning the range of an invariant mean, Proc. Amer. Math. Soc. 26 (1970), 105-107.

4. - On topologically invariant means on a locally compact group, Trans. Amer. Math. Soc. 151 (1970), 443-456.

5. M. M. Day, Amenable semigroups, Illinois J. Math. 1 (1957), 509-544. MR 19, 1067.

6. - Fixed-point theorems for compact convex sets, Illinois J. Math. 5 (1961), 585-590. MR 25 \#1547.

7. L. R. Fairchild, Extreme invariant means and minimal sets in the Stone-Čech compactification of a semigroup, Thesis, University of Illinois, Urbana, Ill., 1970.

8. E. Granirer, On amenable semigroups with a finite-dimensional set of invariant means. I, II, Illinois J. Math. 7 (1963), 32-58. MR 26 \#1744; 1745.

9. M. Jenison, The set of all generalized limits of bounded sequences, Canad. J. Math. 9 (1957), 79-89.

10. V. L. Klee, Jr., Extremal structure of convex sets. II, Math. Z. 69 (1958), 90104. MR 19, 1065.

11. I. Namioka, F申lner's conditions for amenable semi-groups, Math. Scand. 15 (1964), 18-28. MR 31 \#5062.

12. R. A. Raimi, Minimal sets and ergodic measures in $\beta N-N$, Bull. Amer. Math. Soc. 70 (1964), 711-712. MR 29 \#3608.

13. W. Rudin, Averages of continuous functions on compact spaces, Duke Math. J. 25 (1958), 197-204. MR 20 \#4774.

14. A. A. Tempel'man, Ergodic theorems for general dynamic systems, Dokl. Akad. Nauk SSSR 176 (1967), 790-793 =Soviet Math. Dokl. 8 (1967), 1213-1216. MR 36 \#2779.

15. C. Wilde and $\mathrm{K}$. Witz, Invariant means and the Stone-Čech compactification, Pacific J. Math. 21 (1967), 577-586. MR 35 \#3423.

State University of New York at Buffalo, Amherst, New York 14226 